\title{
BMJ
}

\section{Dressings for venous leg ulcers: systematic review and meta-analysis}

\author{
Simon Palfreyman, research nurse/Smith and Nephew Foundation doctoral student, ${ }^{1}$ \\ E Andrea Nelson, reader, ${ }^{2}$ Jonathan A Michaels, professor of vascular surgery ${ }^{1}$
}

${ }^{1}$ Sheffield Vascular Institute, Northern General Hospital, Sheffield S5 7AU

${ }^{2}$ School of Healthcare, University of Leeds, Leeds LS2 9UT

Correspondenceto:SJPalfreyman simon.palfreyman@sth.nhs.uk

doi:10.1136/bmj.39248.634977.AE

\section{ABSTRACT}

Objective To review the evidence of effectiveness of dressings applied to venous leg ulcers.

Design Systematic review and meta-analysis.

Data sources Hand searches of journals and searches of electronic databases, conference proceedings, and bibliographies up to April 2006; contacts with dressing manufacturers for unpublished studies.

Studies reviewed All randomised controlled trials that evaluated dressings applied to venous leg ulcers were eligible for inclusion. Data from eligible studies were extracted and summarised independently by two reviewers using a data extraction sheet. Methodological quality was assessed independently by two reviewers. Results The search strategy identified 254 studies; 42 of these fulfilled the inclusion criteria. Hydrocolloids were no more effective than simple low adherent dressings used beneath compression (eight trials; relative risk for healing with hydrocolloid 1.02, 95\% confidence interval 0.83 to 1.28). For other comparisons, insufficient evidence was available to allow firm conclusions to be drawn. None of the dressing comparisons showed evidence that a particular class of dressing healed more ulcers. Some differences existed between dressings in terms of subjective outcome measures and ulcer healing rates. The results were not affected by the size or quality of trials or the unit of randomisation. Insufficient data were available to allow conclusions to be drawn about the relative cost effectiveness of different dressings.

Conclusions The type of dressing applied beneath compression was not shown to affect ulcer healing. The results of the meta-analysis showed that applying hydrocolloid dressings beneath compression produced no benefit in terms of ulcer healing compared with applying simple low adherent dressings. No conclusive recommendations can be made as to which type of dressing is most cost effective. Decisions on which dressing to apply should be based on the local costs of dressings and the preferences of the practitioner or patient.

\section{INTRODUCTION}

Multilayer compression bandaging has been identified as the gold standard in the treatment of venous leg ulcers. ${ }^{1-3}$ Dressings are usually placed over the ulcer before compression bandages or hosiery are applied, with the intention of promoting healing and preventing the bandages sticking to the wound. However, the evidence of any increased benefit provided by these dressings, which can contribute significantly to the cost of treating a venous leg ulcer, is less clear than for compression.

The range and type of dressings available have increased since the publication of a systematic review of dressings for venous ulcers by Bradley et al in 1999. ${ }^{4}$ Large numbers of different wound dressings are available, with many ways of classifying them-for example, by physical composition or by describing them as passive, active, or reactive. However, the evidence for their use is equivocal. Whether any particular dressing or type of dressing affects the healing of ulcers needs to be established. In addition, many of these dressings are relatively expensive, with a difference of up to six times in unit cost between the more expensive and cheaper dressings. ${ }^{5}$

This study was based on a recently published Cochrane Collaboration review. ${ }^{6}$ We aimed to assess the effectiveness of wound dressings used in the treatment of venous leg ulcers.

\section{METHODS}

We sought to summarise all randomised controlled trials evaluating dressings in the treatment of venous leg ulcers. Two reviewers (SP and EAN) independently assessed trials for suitability; a third reviewer (JAM) arbitrated any disagreements. We excluded trials that included patients with wounds such as arterial and diabetic ulcers, unless the results for patients with venous ulcers were reported separately. We also excluded trials evaluating topical agents and skin grafting. To structure the many comparisons that can be made between dressings, and to avoid potential double counting of comparisons if we simply summarised the interventions by dressing types, we decided in advance to structure our comparisons as illustrated in box 1 .

The primary outcome measure was time to complete ulcer healing or proportion of ulcers completely healed. We excluded composite outcome measures such as "number of ulcers healed or improved."

We identified randomised controlled trials by searching Medline, Embase, and CINAHL, as well as the Cochrane Wounds Group specialised trials register up to April 2006. Box 2 shows details of all the databases searched and search terms used. We also sought grey literature by examining conference proceedings. 


\section{Box 1 Comparisons of dressing types}

Hydrocolloids
Versus foam
Versus alginate
Versus hydrogel
Versus paste
Versus simple/non-adherent dressings
Versus other dressings
Versus other hydrocolloids
Foams
Versus simple/non-adherent dressings
Versus silicone dressings
Versus other foams
Alginate
Versus simple non-adherent dressings
Versus foam
Versus other alginates
Hydrogel
Versus simple/non-adherent dressings
Versus other hydrogel dressings

We placed no restrictions in terms of language or year of publication. We also hand searched key journals, checked citations, and contacted experts in the field of wound care to enquire about ongoing and recently published trials.

Two reviewers acting independently decided on the inclusion or exclusion of trials. A third reviewer (JAM) adjudicated on any disagreements about inclusion or exclusion. We considered trials for inclusion if they were randomised controlled trials evaluating dressings in the treatment of venous leg ulcers. Trials had to report time to complete ulcer healing, proportion of ulcers completely healed, or reduction in area (healing rate). We excluded composite outcome measures that aggregated, for example, healing and improvement. We assessed the quality of the trials on the basis of factors that have been shown to minimise bias and confounding. ${ }^{7}$ These were comparability of treatment groups at baseline; analysis of outcomes on an intention to treat basis, defined as all people who were allocated to a group being analysed in this intervention group regardless of the actual intervention used; completeness of follow-up; and the blinding and objectivity of outcome assessors.

We used Rev Man (4.1) to analyse data. All analysis was on an intention to treat basis, assuming withdrawals and losses to follow-up to be treatment failures. We estimated the relative risk of healing for each study; where similar interventions were compared in similar populations, we then considered using metaanalysis to estimate an aggregate relative risk. We assessed clinical heterogeneity by assessment of populations, concurrent treatment, trial setting, and outcome measures. Statistical heterogeneity was assessed by using the $I^{2}$ test (we considered $I^{2}$ values of $25 \%$ or less to indicate low heterogeneity and values of $75 \%$ or more to indicate high heterogeneity) and the $\chi^{2}$ test (we considered a significance level of $\mathrm{P}<0.1$ to indicate heterogeneity ${ }^{8}$. In the absence of clinical and statistical heterogeneity, we used a fixed effect model. In the absence of clinical heterogeneity but the presence of statistical heterogeneity, we did a random effects meta-analysis. In the presence of clinical heterogeneity, we did a narrative review. A priori, we specified subgroup analyses to examine the robustness of the results on the basis of study size and the presence or absence of compression and allocation concealment.

\section{RESULTS}

We contacted 14 authors about methods, outcomes, and type of wounds, of whom three replied. Figure 1

\section{Box 2 Details of search strategy}

\section{Search terms used in electronic searches}

(Venous Ulcer or Foot Ulcer or Skin Ulcer or Leg Ulcer or Varicose Ulcer) and (Dressing* or Gauze* or Hydrocolloid* or Alginat* or Hydrogel* or Foam or Film) and (Search filter for RCTs)

Conference proceedings examined

- European Conference on Advances in Wound Management 1991-2003

- European Tissue Repair Society (ETRS) Conference 1993-2001

- American Symposia on Advanced Wound Care 19902001

- Symposium on Venous Leg Ulcers 1985

- British/Swedish Angiology Meeting 1991

- Wound Healing and Scarring 1996

- Meeting of the Venous Forum of the Royal Society of Medicine 1999

-31st Annual Wound, Ostomy and Continence Nurses Conference 1999

- Wound Healing Society Educational Symposium 2000

- 7th Annual Conference of the Canadian Association of Wound Care 2001

- 4th Australian Wound Management Association Conference 2002

- Tissue Viability Society Conference 2003

- Ostomy Wound Management 2003

- Vascular Surgical Society of Great Britain and Ireland 2003

- RCN International Nursing Research Conference 2004

- 2nd World Union of Wound Healing Societies' Meeting 2004

- Wound Healing Society 16th Annual Meeting and Exhibition 2006

Journals hand searched

- CARE-Science and Practice 1979-90

- Decubitus 1987-93

- Journal of the European Wound Management Association 2001-April 2006

- Journal of Tissue Viability 1991-April 2006

- Journal of Wound Care 1991-April 2006

- Phlebology 1986-April 2006

- Wound Repair and Regeneration 1993-April 2006

*Truncated 
Citations identified as potentially relevant $(n=254)$

Excluded as non-RCT on basis of abstract $(n=37)$

Citations retrieved for evaluations $(n=217)$

Excluded after evaluation ( $\mathrm{n}=104)$ :

Not randomised controlled trials $(n=43)$

Had populations with ulcers of mixed

aetiology $(\mathrm{n}=36)$

Evaluated topical treatments $(n=6)$

Evaluated skin grafts $(n=5)$

Non-venous wound types $(n=5)$

Compared Unna's Boot (combined

dressing and compression) $(\mathrm{n}=7)$

Evaluated protective skin spray $(n=1)$

Evaluated bandage system $(n=1)$

Citations of RCTs evaluating dressings in venous ulcers $(n=92)$

$\begin{aligned} & \text { Design or reporting flaws }(n=4): \\ & \text { Crossover trials with no reporting of healing } \\ & \text { at crossover point }(n=3) \\ & \text { Use of multiple dressings }(n=1)\end{aligned}$ Citations eligible for inclusion $(n=44)$
RCTs fulfilling inclusion criteria $(n=42)$
Ongary outcome measure identified
for inclusion in review $(n=44)$

Fig 1 Flow chart of identified trials. RCT=randomised controlled trial

shows the stages of the process of meta-analysis using the QUOROM statement. ${ }^{9}$ Tables 1 to 3 show the characteristics of the included and excluded studies and the quality of the included studies.

Of 254 citations initially identified, 44 studies were eligible for inclusion. Two of these trials were ongoing (the VULCAN trial and the HALT trial). ${ }^{\text {w w2 }}$ We therefore included 42 trials (59 citations) involving 3001 participants. Some trials used the limb or ulcer as the unit of randomisation; 3037 ulcers or limbs were included in the trials. Most $(31 / 42,74 \%)$ of the trials had 100 or fewer participants, and 36\% (15/42) had fewer than 50 participants.

Table 2 shows details of the methodological quality of the trials. Trials that we deemed to be of high quality gave clear inclusion/exclusion criteria, described the method of randomisation and blinding of treatment allocation, analysed on an intention to treat basis, had comparable treatment groups at the start of the trial, and used valid outcome measures.

No inclusion criteria were reported for $38 \%$ of studies. Only $11(26 \%)$ trials stated the method of randomisation. ${ }^{\text {w3-w13 }}$ The others merely stated that treatment allocation was "randomised." Most trials reported that the treatment groups were comparable at baseline. Most of the trials $(31 / 42,74 \%)$ reported the total number of ulcers healed during the trial. The remaining $26 \%(11 / 43)$ of trials used only the ulcer healing rate as an outcome measure. The duration of the trials ranged from four weeks to 48 weeks. The mean duration/follow-up was 14 weeks, and the median duration was eight weeks.
We used a random effects model to obtain aggregate outcomes for the relative risk of complete ulcer healing. This method allows for potential heterogeneity of the treatment effect between studies included in the meta-analysis. However, the conclusions from the analysis were the same with both fixed effects and random effects models, even allowing for the variations in precision between the two methods. The scope for subgroup analysis was limited owing to the small number of trials within each comparison. Table 4 shows the results of the meta-analysis.

\section{Hydrocolloid dressings}

Twenty seven trials evaluated hydrocolloid dressings.

\section{Hydrocolloid versus low adherent dressings}

Nine trials (928 participants) compared hydrocolloid and low adherent dressings. ${ }^{\mathrm{w} 4 \mathrm{w} 7 \mathrm{w} 9 \mathrm{w} 10 \mathrm{w} 14-\mathrm{w} 18} \mathrm{We}$ excluded one trial from the meta-analysis, however, as it did not report the number of people whose ulcer had healed. ${ }^{\text {w17 }}$ The remaining eight trials included 792 people, and the pooled relative risk for healing with hydrocolloid was 1.02 (95\% confidence interval 0.83 to 1.25) (fig 2).

We detected significant heterogeneity within this comparison $\left(I^{2}=46.6 \% ; \chi^{2}=13.11, \mathrm{df}=7 ; \mathrm{P}=0.07\right)$. Retrospective exploration of the heterogeneity identified one trial that seemed to differ from the others. ${ }^{\text {w7 }}$ It included only small ulcers (wound area less than 5 $\mathrm{cm}^{2}$ ). Exclusion of this trial removed the statistical heterogeneity and did not affect the finding of no evidence of a difference in healing rate between hydrocolloids and simple low adherent dressings (relative risk $=0.98$, 0.85 to $1.12 ; I^{2}=0 \%$ ).

\section{Hydrocolloid versus foam dressings}

Four trials (311 participants) reported the total number of ulcers healed at 12 weeks for hydrocolloid dressings compared with foam dressings. ${ }^{\text {w5 w19-w21 }}$ Meta-analysis showed a pooled relative risk for healing of 0.98 (0.79 to 1.22 ) (fig 3), indicating no evidence of a statistically significant difference in healing rates. We detected no heterogeneity within the comparison $\left(I^{2}=0 \% ; \chi^{2}=0.07\right.$, $\mathrm{df}=3, \mathrm{P}=0.97)$. One of the trials used the ulcer rather than the patient as the unit of randomisation for the intervention. ${ }^{\text {w5 }}$ Excluding this trial from the analysis did not affect the conclusions of the meta-analysis (relative risk $=0.97,0.74$ to 1.28 ).

\section{Hydrocolloid versus alginate dressings}

Two trials (80 participants) compared hydrocolloid dressings with alginate dressings. ${ }^{\text {w22 w23 }}$ The pooled relative risk for ulcer healing for hydrocolloids compared with alginate dressings was $0.72(0.48$ to 1.69$)$ at 6 -13 weeks. We found high heterogeneity $\left(I^{2}=52 \%\right.$; $\left.\chi^{2}=2.08, \mathrm{df}=1 ; \mathrm{P}=0.15\right)$.

One trial reported cost data and concluded that the cost of materials for hydrocolloids was $£ 67$ cheaper than for alginates. ${ }^{\text {w23 }}$ However, no detail was given of how the costs were calculated, where the data included 


\section{Table 1| Characteristics of included studies}

\section{Reference}

\section{Participants}

Netherlands and Denmark; $n=118$, but 99 included in analysis; duration 8 weeks

Arnold $1994^{\mathrm{w15}} \quad$ UK and USA; $\mathrm{n}=70$; No of ulcers=90; duration 10 weeks

\begin{tabular}{ll}
\hline Backhouse $1987^{\text {w16 }}$ & UK; $n=56$; duration 12 weeks \\
\hline Banerjee $1997^{\text {w31 }}$ & UK; $n=71$; duration 17 weeks \\
\hline Banks $1996^{\text {w20 }}$ & UK; $n=100$; duration 13 weeks \\
\hline Blair $1988^{\text {w4 }}$ & $\begin{array}{l}\text { UK; } n=120 \text { "consecutive ulcers," with } 60 \text { in each } \\
\text { stage; duration } 12 \text { weeks }\end{array}$ \\
\hline
\end{tabular}

Bowszyc $1995^{\text {w5 }}$

Callam $1992^{\text {w32 }}$ Poland; $\mathrm{n}=80$ (82 limbs); duration 12 weeks

Caprio $1994^{\text {w29 }}$
Andersen $2002^{\mathrm{w} 3}$
Dressing

I1: $\mathrm{FD}(\mathrm{n}=58 ; 53$ included in analysis); 12: $\mathrm{FD}(\mathrm{n}=60 ; 46$ included in analysis)

11: HC ( $n=35) ; 12:$ standard dressing $(n=35)$

11: HC ( $(n=28) ;$ 12: LA ( $(n=28)$

I1: FD ( $n=36) ; 12:$ LA ( $n=35)$

I1: HC ( $n=50) ; 12: F D(n=50)$

I1: HC; 12: Flamazine; control: LA

11: HC ( $(n=40) ; 12: F D(n=40)$

I1: FD ( $n=66) ;$ 12: LA ( $n=66)$

11: HC (n=49); 12: LCD ( $n=49)$
Outcomes reported

No healed-I1: 18/53 (34\%; IT 18/58, 31\%); |2: 18 46 (39\%; IT 18/60, 30\%). Mean time to healing-11 5.2 (SD 1.9) weeks; I2: 5.0 (SD 1.7) weeks

No healed-11: 11/35 (31\%); 12: 14/35 (40\%). Mean reduction in ulcer area-11: 71\% (SE 4.3); 12: 43\% (SE 7.1)

No healed-11: 21/28 (75\%); 12: 22/28 (78\%) No healed-11: $11 / 36$ (30.5\%); 12: 8/35 (23.0\%)

No healed-11: 19/50 (38\%); 12: 18/50 (36\%)

No healed-11: HC 22/30 (73\%), LA 23/30 (77\%); 12: Flamazine 19/30 (63\%), LA 24/30 (78\%)

No healed-I1: 24/40 (60\%); 12: 24/40 (60\%)

No healed-11: 31/66 (47\%); 12: (23/66 (35\%) $(P=0.08)$

No healed-11: 25/49 (51\%); 12: 20/49 (41\%).

Reduction in wound area ( $\mathrm{mm}^{2} /$ week) - $11: 152.7 ; 12$ 103.66. Total mean cost (lira) dressing materials per patient ( $£=2400$ lira)-11: 102607 (£42.75); 12: 142 527 (£59.37)

Charles 2002 $2^{\text {w19 }} \quad$ UK; $n=91$; duration 12 weeks $\quad$ I1: FD ( $\left.n=31\right) ; 12: H C(n=31) ; 13: H C(n=29)$

Part 1-11:0.9\% normal saline; 12: HG; no detail of numbers provided for groups. Part 2-11: porcine skin $(n=11)$; 12: aluminium foil dressing $(n=20)$; 13: zinc oxide and Tensoplast bandage $(\mathrm{n}=13)$

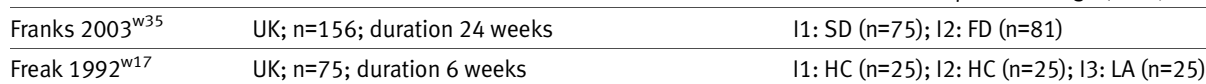

\begin{tabular}{|c|c|c|}
\hline Greguric $1994^{\text {w30 }}$ & Croatia; $n=110$; duration "10 dressing changes" & 11: standard dressing $(n=55) ; 12: \mathrm{HC}(n=55)$ \\
\hline Groenwald $1984^{\text {w6 }}$ & South Africa; $n=72$; duration 8 weeks & 11: conventional treatment $(n=36) ; 12: \mathrm{HC}(\mathrm{n}=36)$ \\
\hline Grotewohl $1994^{\text {w27 }}$ & $\begin{array}{l}\text { Germany; } n=84 \text {, but only } 63 \text { participants reported; } \\
\text { duration } 28 \text { days }\end{array}$ & I1: HG (n=39); I2: HC (n=24) \\
\hline Hansson $1998^{\text {w18 }}$ & $\begin{array}{l}\text { Sweden/Denmark/Netherlands/UK; n=153; duration } \\
12 \text { weeks }\end{array}$ & I1: HG (n=56); I2: HC ( $n=48) ; 13:$ LA ( $(n=49)$ \\
\hline Hornemann $1987^{\text {w37 }}$ & Germany; n=148; duration 4 weeks & I1: HG (n=73); I2: HG (n=75) \\
\hline Limova $1996^{\text {w24 }}$ & USA; $n=31$; duration 8 weeks & I1: HC (n=17); I2: HC (n=14) \\
\hline Limova $2003^{\text {w40 }}$ & $\begin{array}{l}\text { USA; } n=20 \text {, but only } 19 \text { included in analysis; duration } \\
6 \text { weeks }\end{array}$ & I1: AD (n=10); I2: AD (n=9) \\
\hline Lindholm $1994^{\text {w28 }}$ & Sweden; $n=28$; duration 6 weeks & I1: GD (n=14); I2: HC (n=14) \\
\hline Meredith $1988^{\mathrm{w} 7}$ & $\begin{array}{l}\text { UK; } n=50 \text {, but one excluded from analysis; duration } \\
6 \text { weeks }\end{array}$ & I1: HC (n=25); I2: LA (n=24) \\
\hline Moffatt $1992^{\text {w39 }}$ & UK; $n=60$; duration 12 weeks & I1: AD (n=30); I2: LA (n=30) \\
\hline Moffatt $1992^{\text {w9 }}$ & UK; $n=60$; duration 12 weeks & I1: HC (n=30); I2: LA ( $(n=30)$ \\
\hline Mulder $1995^{\text {w34 }}$ & UK; $n=40$ (39 reported); duration 16 weeks & I1: FD (n=19); I2: AD (n=20) \\
\hline Mulligan $1986^{\text {w8 }}$ & UK; n=101 (97 reported); duration 6 weeks & $\begin{array}{l}\text { 11: knitted fabric charcoal dressing }(n=65) ; 12: \\
\text { conventional treatment decided by physician }(n=35)\end{array}$ \\
\hline Nelson $1995^{\text {w10 }}$ & UK; $n=200$; duration 24 weeks & I1: LA (n=98); 12: HC (n=102) \\
\hline Ormiston $1985^{\text {w11 }}$ & UK; $n=61$; duration 12 weeks & $\begin{array}{l}\text { I1: } \mathrm{HG} \text { and Melolin dressing }(n=30 ; 1 T=31) ; 12 \text { : } \\
\text { standard treatment (gentian violet, Polyfax salmyxin, } \\
\text { and bacitracin ointment plus gauze pad) }(n=30)\end{array}$ \\
\hline Pessenhoffe & $8 ; \mathrm{d}$ & I1: FD ( $n=25) ; 12:$ LA $(n=23)$ \\
\hline
\end{tabular}

Scurr 1994 1 W22 $\quad$ I1: $A D(n=20) ; 12: H C(n=20)$

Skog $1983^{\text {w42 }}$ Sweden and Norway; $\mathrm{n}=95$ (74 reported); duration 6 weeks

Smith $1994^{\text {w23 }} \quad$ UK; n=40; duration 6 weeks

11: HG ( $n=38) ;$ 12: standard treatment $(n=36)$

I1: $\mathrm{HC}(\mathrm{n}=22) ; \mathrm{I2}: \mathrm{AD}(\mathrm{n}=18)$
No healed-|1: 18/31 (58\%); |2:17/31 (55\%); |3: $17 \mid$ 29 (57\%)

Part 1-ulcer healing rate, states "no significant difference" between groups. Part 2-mean reduction in wound area, $11: 70 \% ; 12: 10 \% ; 13: 80 \%$

No healed-11: 50/75 (66.7\%); 12: 50/81 (61.7\%)

Reduction in mean ulcer area $\left(\mathrm{cm}^{2}\right)-11: 2.98$ (SE 0.44); 12: 2.09 (SE 0.51); 13: 4.31 (SE 0.64); P>0.05. Median time to complete healing (life table)-11: 6 . 85 weeks; 12: 4.25 weeks; 13: 6.17 weeks

No healed-11: $0 ; 12: 3$. Mean reduction in ulcer area $\left(\mathrm{mm}^{2} /\right.$ day $)-11: 21 ; 12: 32$

Reduction in ulcer size-11: 22.62\%; 12: 67.64\%; $\mathrm{P}<0.001$

Reduction in ulcer area-11: 44.6\%; 12: 33.3\%. Mean reduction in ulcer surface area- $11: 4.5 \mathrm{~cm}^{2} ; 12: 2.1 \mathrm{~cm}^{2}$ No healed-11: 8/56 (14\%); 12: 5/48 (10\%); 13: 7/49 (14\%)

Median wound reduction-11: 50\%; 12: 20\% No healed-11: 10/17 (60\%); 12: 2/14 (14\%) No healed-11: 0/10 (0\%); 12: 2/9 (22\%)

Reduction in wound area-11: 19\%; 12: 51\% No healed-11: 19/25 (76\%); 12: 6/24 (25\%). Total area healed $\left(\mathrm{cm}^{2}\right)-11: 21.1 ; 12: 7.7$. Cost of dressing_ I1:£436.86; 12; £855.87

No healed-11: 26/30 (87\%); 12: 24/30 (80\%)

No healed-11: $13 / 30$ (43\%); 12: 7/30 (23\%)

No healed-11: 10/19 (53\%); 12: 6/20 (30\%)

Mean ulcer reduction-11:28.7\%; 12:11.7\%. Mean ulcer healing rate/week-11: $1.2 \mathrm{~cm} ; 12: 0.2 \mathrm{~cm}$ No healed-11: 44/98 (45\%); 12: 49/102 (48\%) No healed-I1: 12/31 (39\%); 12: 7/30 (23\%). Healing rate per week $\left(\mathrm{cm}^{2}\right)$-11: 0.89 (SE 0.1); 12: 0.46 (SE $0.1) ; \mathrm{P}=0.0001$

Relative change in ulcer size-11: decrease $65.6 \%$ (SD 47.0); 12: increase 78.3\% (SD 215.8)

No healed-11: 6/20 (30\%); 12: 2/20 (10\%)Mean reduction in area-11: $90.7 \%$ (SD 14.5); 12: 80.2\% (SD 22.4)

Mean reduction in ulcer area-11: decrease of 34\%; 12 : increase of $5 \%$

No healed-11:4/22 (18\%); 12:2/18 (11\%). Change in ulcer area-11:57.1\%; 12:34.9\%. Cost of treatmentI1: £431.73; 12: £364.08 
No healed-I1, small HC: 38/64 (56\%); I2, small LA: 43/62 (69\%); I1, large HC: $12 / 35(34 \%) ; ~ I 2$, large LA: 4/39 (10\%). Cost of treatment-l1, small: $£ 48.96 ; 12$ small: £39.95; I1, large: £526.63; 12, large: £183.75

\begin{tabular}{lll}
\hline Stacey $1997^{\text {w41 }} \quad$ Australia; $n=113$, with 133 limbs; duration 36 weeks & $\begin{array}{l}\text { I1: zinc oxide ointment impregnated woven cotton } \\
\text { bandage (Viscopaste bandage); I2: zinc oxide } \\
\text { ointment impregnated knitted stockinet (Acoband); } \\
\text { I3: AD (Kaltostat) }\end{array}$
\end{tabular}

Taddeucci $2004^{\text {w43 }} \quad$ Italy; $\mathrm{n=17}$, with 24 ulcers; duration 8 weeks $\quad$ I1: hyaluronan derivative fleece dressing ( $\mathrm{n}=12$
ulcers); I2: $L A$ ( $n=12$ ulcers)

Tarvainen $1988^{\text {w13 }} \quad$ Finland; $n=27$; duration 8 weeks
I1: $H G(n=14) ; 12: H G(n=13)$ No healed-11: Viscopaste 34/43 (86\%); 12: Acoband 26/44 (66\%); 13: Kaltostat 26/46 (57\%)

\begin{tabular}{lll}
\hline Veraart 1994 $^{\text {w25 }}$ & Netherlands; $\mathrm{n}=38 ;$ duration 8 weeks & I1: HC (Comfeel extra-absorbing dressing \\
& & HC (Granuflex/Duoderm CGF) $(\mathrm{n}=19)$
\end{tabular}

Vin 2002 ${ }^{\text {w36 }} \quad$ France; $n=73$; duration 12 weeks I1: HG $(n=37) ; 12:$ LA $(n=36)$

\begin{tabular}{ll}
\hline Vincent $^{\text {w26 }}$ & UK; $n=29 ;$ duration 6 weeks \\
\hline Weiss $1996^{\text {w33 }}$ & USA; $n=18 ;$ duration 16 weeks \\
\hline Wunderlich 1991w44 & $\begin{array}{l}\text { Germany; } n=40, \text { of which } 38 \text { produced "evaluable" } \\
\text { data; duration } 6 \text { weeks }\end{array}$
\end{tabular}

I1: HC (n=16); I2: HC ( $n=13)$

I1: FD ( $=10)$; 12: FD $(n=8)$

I1: 5 days' cleaning with enzymatic debrider and then application of polyamide active charcoal dressing with $0.15 \%$ silver; 12 : 5 days' cleaning using mechanical and enzymatic debridement and then dressing according to stage of healing. Granulation=paraffin oil or PVI (polyvinyl iodine) cream; epithelialisation=Fettgaze or oil in water emulsion

Zuccarelli $1992^{\text {w21 }} \quad$ France; $n=40$, but only 38 analysed; duration
I1: FD ( $n=19) ;$ I2: HC ( $=19)$ No healed—I1: 9/19 (47\%); 12: 9/19 (47\%)

No healed-11: 2/12 (16\%); |2: 1/12 (8\%). Reduction in ulcer area $\left(\mathrm{cm}^{2}\right)-11: 8.1(\mathrm{n}=12) ; 12: 0.4(\mathrm{P}<0.002)$

No healed-I1: $7 / 14$ (50\%); 12: 5/13 (39\%)

No healed-I1: 18/37 (49\%); 12: 12/36 (33\%). Mean reduction in wound area-11: $54.4 \%$ (SE 10); 12: $36.5 \%$ (SE 11.4). Median reduction in wound area-11: $82.4 \%$; $12: 44.6 \%$

Reduction in wound area-11: 45\%; 12: $57 \%$

No healed-11: 8/10 (80\%); I2: 4/8 (50\%). Mean time to healing-11: 5.6 weeks; $12: 6.5$ weeks

No healed-I1: $6 / 19$ (31.6\%, IT=30\%); I2: 2/20 $(10.5 \%, I T=10 \%)$. Reduction in wound area-I $60 \% ; 12: 75 \%$

intervention 3; ITT=intention to treat; $L A=$ low adherent

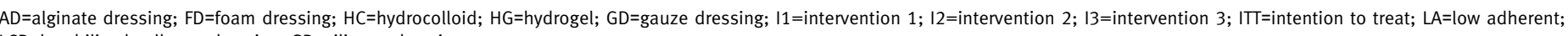
$\mathrm{LCD}=$ lyophilised collagen dressing; $\mathrm{SD}=$ silicone dressing.

in the costs came from (from patients or from drug tariff data), or the type and extent of discounting used.

\section{Hydrocolloid versus hydrocolloid}

Three trials (98 participants) compared various hydrocolloid dressings. ${ }^{\text {w24-w26 }}$ We could include only two in the meta-analysis, as one did not report the total number of ulcers healed. ${ }^{\text {w26 }}$ The meta-analysis showed high heterogeneity $\left(I^{2}=69.7 \% ; \chi^{2}=3.3, \mathrm{df}=1 ; \mathrm{P}=0.07\right)$ and no statistical difference between the dressings (relative risk=1.56, 0.67 to 3.63 ).

\section{Hydrocolloid versus other dressings}

Two trials (237 participants) compared hydrocolloid and hydrogel dressings. ${ }^{\text {w18 w27 }}$ One trial allocated treatment "according to the principle of random selection." ${ }^{\text {w27 }}$ The analysis was not intention to treat. The outcomes reported were percentage reduction in ulcer area at 28 days $(44.6 \%$ hydrogel $v 33.3 \%$ hydrocolloid) and the reduction of the ulcer surface area at 28 days $\left(4.5 \mathrm{~cm}^{2}\right.$ hydrogel $v 2.1 \mathrm{~cm}^{2}$ hydrocolloid). The total number of ulcers healed in each group was not reported. The other trial had three arms and was also included in the hydrocolloid versus low adherent dressings comparison. ${ }^{\text {w18 }}$

One trial (28 participants) compared hydrocolloid and gauze. ${ }^{\text {w28 }}$ It reported a relative reduction in ulcer area of 19\% for the gauze group and $51 \%$ for the hydrocolloid group. No statistical analysis of the results was reported.

One trial (93 participants with 98 ulcers) compared hydrocolloid dressing (Duoderm E) and a lyophilised collagen dressing. ${ }^{\text {w29 }}$ The unit of randomisation was the ulcer and not the patient. No statistical differences between the dressings were reported.

One trial (110 participants) compared hydrocolloid dressing (Varihesive E) and magnesium sulphate paste beneath a gauze dressing. ${ }^{\text {w30 }}$ The duration of the trial was for 10 dressing changes, but no details were given on trial length. The trial reported three ulcers healed in the hydrocolloid group and no ulcers healed in the other group.

\section{Foam dressings}

\section{Foam versus low adherent dressings}

Three trials (253 participants) compared foam dressings with low adherent dressings. ${ }^{\text {w12 w31 w32 One trial }}$ did not report the total number of ulcers healed and so could not be included in the meta-analysis. ${ }^{\text {w12 }}$ For the other two studies, the pooled relative risk was 1.35 (0.93 to 1.94$)$, with no statistical heterogeneity $\left(I^{2}=0 \% ; \chi^{2}=0.0, \mathrm{df}=1 ; \mathrm{P}=0.99\right)$.

\section{Foam versus foam dressings}

Two trials (136 participants) compared various foam dressings. ${ }^{\text {w3 } 33}$ The aggregate relative risk of healing for the two studies was $1.2(0.77$ to 1.87$)$, with no statistical heterogeneity $\left(I^{2}=0 \%, \chi^{2}=0.88, \mathrm{df}=1 ; \mathrm{P}=0.35\right)$.

\section{Foam versus alginate dressings}

One trial (40 participants) compared foam dressings with alginate dressings. ${ }^{\text {w34 }}$ No statistically significant difference was found between the dressings. The reported hazard ratio for healing was 1.75 (0.79 to 3.88). 
Table 2 | Methodological quality of included studies

\begin{tabular}{|c|c|c|c|c|c|c|c|}
\hline Study & $\begin{array}{l}\text { Baseline } \\
\text { comparability }\end{array}$ & $\begin{array}{l}\text { Inclusion and } \\
\text { exclusion } \\
\text { criteria }\end{array}$ & $\begin{array}{l}\text { Method of } \\
\text { randomisation }\end{array}$ & $\begin{array}{l}\text { Allocation } \\
\text { concealment }\end{array}$ & $\begin{array}{l}\text { Sample size } \\
\text { calculation }\end{array}$ & ITT & $\begin{array}{l}\text { Blinding of } \\
\text { outcome }\end{array}$ \\
\hline Andersen $2002^{\mathrm{w} 3}$ & Yes & Yes & Yes & Adequate & No & No & Unclear \\
\hline Arnold $1994^{\mathrm{w} 15}$ & Yes & Yes & No & Unclear & No & Yes & Unclear \\
\hline Backhouse $1987^{\text {w16 }}$ & Yes & No & No & Unclear & No & Yes & Unclear \\
\hline Banerjee $1997^{\text {w31 }}$ & No & Yes & No & Unclear & No & Yes & Unclear \\
\hline Banks $1996^{\text {w20 }}$ & Yes & Yes & No & Unclear & No & Yes & Unclear \\
\hline Blair $1988^{w 4}$ & Yes & Yes & Yes & Adequate & No & Yes & Unclear \\
\hline Bowszyc $1995^{\text {w5 }}$ & Yes & Yes & Yes & Unclear & No & No & Unclear \\
\hline Callam $1992^{\text {w32 }}$ & Yes & Yes & No & Adequate & No & Yes & Yes \\
\hline Caprio $1994^{\text {w29 }}$ & No & Yes & No & Unclear & No & Yes & Unclear \\
\hline Charles $2002^{\text {w19 }}$ & Yes & Yes & No & Unclear & No & Yes & Unclear \\
\hline Eriksson $1984^{\text {w38 }}$ & Yes & No & No & Unclear & No & No & Unclear \\
\hline Franks $2003^{\text {W35 }}$ & No & Yes & No & Unclear & No & Yes & Unclear \\
\hline Freak $1992^{\text {w17 }}$ & Yes & No & No & Unclear & No & Yes & Unclear \\
\hline Greguric $1994^{\text {w30 }}$ & Yes & No & No & Unclear & No & Yes & Unclear \\
\hline Groenwald $1984^{\text {w6 }}$ & No & No & Yes & Unclear & No & Yes & Unclear \\
\hline Grotewohl $1994^{\text {w27 }}$ & No & No & No & Unclear & No & No & Unclear \\
\hline Hansson $1998^{\text {w18 }}$ & No & Yes & No & Unclear & No & Yes & Unclear \\
\hline Hornemann $1987^{\text {w37 }}$ & No & No & No & Unclear & No & Yes & Unclear \\
\hline Limova $1996^{\text {w24 }}$ & Yes & Yes & No & Unclear & No & Yes & Unclear \\
\hline Limova $2003^{\text {w40 }}$ & No & No & No & Unclear & No & No & Unclear \\
\hline Lindholm $1994^{\text {w28 }}$ & No & Yes & No & Unclear & No & Yes & Unclear \\
\hline Meredith $1988^{\mathrm{w} 7}$ & Yes & Yes & Yes & Unclear & No & No & Unclear \\
\hline Moffatt $1992^{\text {w39 }}$ & Yes & Yes & No & Unclear & No & Yes & Unclear \\
\hline Moffatt $1992^{\text {w9 }}$ & Yes & Yes & Yes & Unclear & Yes & Yes & Unclear \\
\hline Mulder $1995^{\text {w34 }}$ & No & No & No & Unclear & No & No & Unclear \\
\hline Mulligan $1986^{\text {w8 }}$ & Yes & Yes & Yes & Unclear & No & No & Unclear \\
\hline Nelson $1995^{\mathrm{w} 10}$ & Yes & Yes & Yes & Adequate & Yes & Yes & Unclear \\
\hline Ormiston $1985^{\mathrm{w} 11}$ & Yes & No & Yes & Adequate & No & No & Unclear \\
\hline Pessenhoffer $1992^{\text {w12 }}$ & No & No & Yes & Unclear & No & Yes & Unclear \\
\hline Scurr $1994^{\text {w22 }}$ & Yes & Yes & No & Unclear & No & Yes & Unclear \\
\hline Skog $1983^{\text {w42 }}$ & Yes & No & No & Unclear & No & No & Unclear \\
\hline Smith $1994^{\text {w23 }}$ & Yes & No & No & Unclear & No & Yes & Unclear \\
\hline Smith $1992^{\text {w14 }}$ & Yes & Yes & No & Unclear & No & Yes & Unclear \\
\hline Stacey $1997^{\text {w41 }}$ & Yes & Yes & No & Unclear & No & Yes & Unclear \\
\hline Taddeucci $2004^{\text {w43 }}$ & No & No & No & Unclear & No & Yes & Unclear \\
\hline Tarvainen $1988^{\text {w13 }}$ & Yes & Yes & Yes & Adequate & No & Yes & Unclear \\
\hline Veraart $1994^{\mathrm{w} 25}$ & Yes & Yes & No & Unclear & No & Yes & Unclear \\
\hline Vin $2002^{\text {w36 }}$ & Yes & Yes & No & Unclear & No & Yes & Unclear \\
\hline Vincent $^{\text {w26 }}$ & Yes & No & No & Unclear & No & Yes & Unclear \\
\hline Weiss $1996^{\text {w33 }}$ & No & No & No & Unclear & No & Yes & Unclear \\
\hline Wunderlich $1991^{\text {w44 }}$ & Yes & Yes & No & Unclear & No & No & Unclear \\
\hline Zuccarelli $1992^{\text {w21 }}$ & Yes & Yes & No & Unclear & No & No & Unclear \\
\hline
\end{tabular}

Foam versus silicone dressings

One trial (156 participants) compared foam dressings against silicone dressings. ${ }^{\mathrm{w} 35}$ No statistically significant difference was found between the dressings. The reported hazard ratio for healing was 1.17 (0.79 to 1.72).

\section{Hydrogel dressings}

Hydrogel versus low adherent dressings

Two trials (151 participants) compared hydrogel dressings with low adherent dressings. ${ }^{\text {w11 w36 }}$ The aggregate relative risk for healing with hydrogel compared with low adherent dressings was 1.53 (0.96 to $2.42 ; I^{2}=0 \%$, $\chi^{2}=0.07, \mathrm{df}=1 ; \mathrm{P}=0.79$ ).

Hydrogel versus hydrogel

Two trials (175 participants) compared different hydrogels. ${ }^{\text {w13 w37 }}$ However, we were unable to do a meta-analysis as only one trial reported the total number of ulcers healed, and this found no statistically significant difference between the two groups. ${ }^{\text {w13 }}$ 
Table 3 | Reasons for exclusion of studies

Study

Agren $1990^{\text {w45 }}$

Alcaraz $2003^{\mathrm{w} 46}$

Alicandro $2003^{\mathrm{w4} 7}$

Armstrong $1995^{\text {w48 }}$

Armstrong $1997^{\text {w49 }}$

Bale 1994w50

Bale 1998 w51

Ballard 2002 ${ }^{\mathrm{w} 52}$

Banks $1995^{\text {w53 }}$

Banks $1997^{\text {w54 }}$

Barnett 1988w55

Bartoletti $1997^{\text {w56 }}$

Berry $1993^{\text {w57 }}$

Bianchi 2001 ${ }^{\text {w5 }}$

Bonnetblanc 2004 ${ }^{\mathrm{w5} 9}$

Brandrup 1990w60

Brown-Etris 2004 ${ }^{\mathrm{w} 61}$

Bull $1995^{\mathrm{w6} 2}$

Burgess $1993^{\text {w63 }}$

Burgos $1989^{\text {w64 }}$

Capillas 2000 w65

Casoni $2002^{\text {w66 }}$

Chaloner 1992 ${ }^{\text {w67 }}$

Chaloner 1996w68

Charles 2004 ${ }^{\text {w69 }}$

Cherry $1996^{\text {w70 }}$

Cherry 2001 ${ }^{\text {w71 }}$

Colletta $2003^{\text {w72 }}$

Collier 1992 ${ }^{\text {w73 }}$

Cordts $1992^{\text {w74 }}$

Creese $1986^{\text {w75 }}$

Daniels $2002^{\text {w76 }}$

Davis $1992^{\text {w77 }}$

Diem $1987^{\mathrm{w78}}$

Dmochowska 1999w79

Egan $1983^{\text {w80 }}$

Eriksson $1986^{\text {w81 }}$

Falanga $1998^{\text {w82 }}$

Farina $1997^{\text {w83 }}$

Fivenson $2003^{\text {w84 }}$

Floden $1978^{\text {w85 }}$

Frank $1979^{\text {w86 }}$

Franken $1999^{\text {w87 }}$

Franks $1993^{\text {w88 }}$

Friedman $1984^{\text {w89 }}$

Gamborg-Nielson $1989^{\text {w90 }}$

Gibson $1985^{\text {w91 }}$

Goldman $2003^{\text {w92 }}$

Halbert $1992^{\text {w93 }}$

Handfield-Jones $1988^{\text {w9 }}$

Harcup $1986^{\text {w95 }}$

Hart $1998^{\text {w96 }}$

Hermans $2000^{\text {w97 }}$

Hoffman $2000^{\text {w98 }}$

Holloway $1989^{\text {w99 }}$

Hutchinson $1992^{\text {w100 }}$
Reason for exclusion

Includes ulcers of mixed aetiology; no reporting by ulcer type

Non-randomised study

Includes ulcers of mixed aetiology; no reporting by ulcer type

Includes ulcers of mixed aetiology; no reporting by ulcer type

Includes ulcers of mixed aetiology; no reporting by ulcer type

Includes arterial ulcers, with no separate reporting by ulcer type

Includes ulcers of mixed aetiology; no reporting by ulcer type

Non-randomised study

No reporting of ulcer healing

Includes ulcers of mixed aetiology; no reporting by ulcer type

Review paper

No reporting of ulcer healing

Evaluation of dressings for pilonidal sinus

Review article and case study

Evaluation of protective spray not dressing

Includes arterial ulcers with no separate reporting by ulcer type

No reporting of ulcer healing

No reporting of ulcer healing

No reporting of ulcer healing

Evaluation of topical growth factors applied via dressings

Reports cost to heal $1 \mathrm{~cm}^{2}$ ulcer; authors unable to provide more data

Evaluation of compression

Includes ulcers of mixed aetiology; no reporting by ulcer type

Did not report healing outcomes

Non-randomised study

Study examining topical application

Evaluation of topical application

Non-randomised study

Includes ulcers of mixed aetiology; no reporting by ulcer type

Evaluates compression

Non-randomised study

Non-randomised study

Evaluates compression

Non-randomised study

No reporting of total number of ulcers healed or ulcer healing rates

Intervention is preparation for skin grafting; does not report healing

Evaluates double layer bandage system

Evaluates skin graft

Does not report ulcer healing

Non-randomised study

Does not report ulcer healing

Does not report ulcer healing

Description of trial methods; no results

Letter; does not report outcome data

Non-randomised study

Includes ulcers of mixed aetiology; no reporting by ulcer type

Arterial ulcers only

Non-randomised study

Non-randomised study

Crossover study with no report of ulcer healing at crossover point

No breakdown of numbers randomised to each group

No breakdown of numbers allocated to groups; no reply to query

Includes ulcers of mixed aetiology; no reporting by ulcer type

Non-randomised study

No data on ulcer healing

Outcome measure "healed or improved;" no reply from author 
Jasiel $1996^{\mathrm{w} 101}$

\begin{tabular}{|c|c|}
\hline Johnson $1992^{\text {w102 }}$ & Study of dressings for pressure sores \\
\hline Jones $2003^{\text {w103 }}$ & No report of healing rates or total number of ulcers healed \\
\hline Kalis $1993^{\text {w104 }}$ & Includes ulcers of mixed aetiology; no reporting by ulcer type \\
\hline Kammerlander $2000^{\text {w105 }}$ & Includes ulcers of mixed aetiology; no reporting by ulcer type \\
\hline Karlsmark $2003^{\text {w106 }}$ & Non-randomised study \\
\hline Kero $1987^{\text {w107 }}$ & Non-randomised study \\
\hline Kerstein $2000^{\text {w108 }}$ & Non-randomised study \\
\hline Kikta $1988^{\text {w109 }}$ & Evaluation of compression \\
\hline Koksal $2003^{\text {w110 }}$ & Evaluation of compression \\
\hline Kucharzewski $2003^{\text {w111 }}$ & Evaluation of topical treatments \\
\hline Lansdown $2003^{\text {w112 }}$ & Non-randomised study \\
\hline Larsen $1995^{\text {w113 }}$ & No reporting of ulcer healing \\
\hline Larsen $1997^{\text {w114 }}$ & No reporting of ulcer healing \\
\hline Larsen $2001^{\text {w115 }}$ & No reporting of ulcer healing \\
\hline Laudanska $1988^{\text {w116 }}$ & Study endpoint combined complete healing and "very superficial" \\
\hline Leaper $1991^{\text {w117 }}$ & Includes ulcers of mixed aetiology; no reporting by ulcer type \\
\hline Lindholm $1993^{\mathrm{w118}}$ & Includes ulcers of mixed aetiology; no reporting by ulcer type \\
\hline Lindholm $1995^{\text {w119 }}$ & No reporting of ulcer healing \\
\hline Ljunberg $1998^{\text {w120 }}$ & Includes ulcers of mixed aetiology; no reporting by ulcer type \\
\hline Llewellyn $1995^{\text {w121 }}$ & Non-randomised study \\
\hline Loiterman $1991^{\text {w122 }}$ & Non-randomised study \\
\hline Margolis $1993^{\mathrm{w} 123}$ & Non-randomised study \\
\hline Mayrovitz $1992^{\text {w124 }}$ & Includes ulcers of mixed aetiology; no reporting by ulcer type \\
\hline McMullen $1991^{\text {w125 }}$ & Non-randomised study \\
\hline Meaume $2002^{\mathrm{w} 126}$ & Non-randomised study \\
\hline Meaume $2004^{\mathrm{w} 127}$ & No reporting of ulcer healing \\
\hline Mekkes $1992^{\text {w128 }}$ & No reporting of ulcer healing \\
\hline Mian $1992^{\text {w129 }}$ & Includes ulcers of mixed aetiology; no reporting by ulcer type \\
\hline Milward $1991^{\text {w130 }}$ & Includes ulcers of mixed aetiology; no reporting by ulcer type \\
\hline Moody $2003^{\text {w131 }}$ & No reporting of ulcer healing \\
\hline Mulder $1993^{\text {w132 }}$ & No reporting of ulcer healing \\
\hline Mulder $1994^{\text {w133 }}$ & States "statistically significant" differences but no results reported \\
\hline Nowak $1995^{\text {w134 }}$ & No reporting of ulcer healing \\
\hline Nyfors $1982^{\text {w135 }}$ & Includes ulcers of mixed aetiology; no reporting by ulcer type \\
\hline Ohlsson $1994^{\mathrm{w} 136}$ & Includes ulcers of mixed aetiology; no reporting by ulcer type \\
\hline Palmeri $1992^{\text {w137 }}$ & Comparing topical agents \\
\hline Perez $2000^{\text {w138 }}$ & No reporting of ulcer healing \\
\hline Petres $1993^{\text {w139 }}$ & No reporting of ulcer healing \\
\hline Polignano $2002^{\text {w140 }}$ & No reporting of ulcer healing \\
\hline Poole $1994^{\mathrm{w141}}$ & No reporting of ulcer healing \\
\hline Poskitt $1987^{\text {w142 }}$ & Study of skin grafts. \\
\hline Price $2003^{\mathrm{w1} 43}$ & Non-randomised study \\
\hline Price $2004^{\mathrm{w} 144}$ & No reporting of ulcer healing \\
\hline Rainey $1993^{\text {w145 }}$ & No reporting of ulcer healing \\
\hline Reynolds $2004^{\mathrm{w} 146}$ & Reports "ulcer improved or healed" \\
\hline Robinson $1993^{\text {w147 }}$ & No reporting of ulcer healing \\
\hline Robinson $1995^{\text {w148 }}$ & Non-randomised study \\
\hline Robinson $1996^{\text {w149 }}$ & Report of trial design \\
\hline Robinson $1997^{\mathrm{w} 150}$ & Includes ulcers of mixed aetiology; no reporting by ulcer type \\
\hline Robledillo $2002^{\text {w151 }}$ & Non-randomised study \\
\hline Rubin $1990^{\text {w152 }}$ & Evaluation of compression \\
\hline Rundle $1981^{\text {w153 }}$ & No reporting of ulcer healing \\
\hline Russell $2004^{\mathrm{w1} 54}$ & Reports only composite outcome of "ulcer healed or improved" \\
\hline Samson $1992^{\text {w155 }}$ & No reporting of ulcer healing; no reply to query \\
\hline Samson $1993^{\text {w156 }}$ & Non-randomised study \\
\hline Sayag $1996^{\text {w157 }}$ & Trial of treatments for pressure ulcers \\
\hline Scalise $2003^{\text {w158 }}$ & Report of trial design \\
\hline
\end{tabular}




\begin{tabular}{|c|c|}
\hline Schmutz $1996^{\text {w159 }}$ & Report of trial design \\
\hline Schulze $2001^{\text {w160 }}$ & No reporting of ulcer healing \\
\hline Scurr $1993^{\text {w161 }}$ & Non-randomised study \\
\hline Serafica $2003^{\text {w162 }}$ & Non-randomised study \\
\hline Sibbald $2004^{\text {w163 }}$ & No reporting of ulcer healing \\
\hline Sikes $1985^{\text {w164 }}$ & Evaluation of compression \\
\hline Sironi $1993^{\mathrm{w} 165}$ & No reporting of ulcer healing \\
\hline Sironi $2003^{\text {w166 }}$ & No reporting of ulcer healing \\
\hline Skene $1992^{\text {w167 }}$ & Reports development of prognostic index to predict time to healing \\
\hline Slezak 2004 ${ }^{\text {w168 }}$ & Non-randomised study \\
\hline Smith $1993^{\text {w169 }}$ & Duplicate publication of Smith $1994^{\text {w23 }}$ \\
\hline Smith $2003^{\text {w170 }}$ & Non-randomised study \\
\hline Stewart $1987^{\mathrm{w171}}$ & Includes ulcers of mixed aetiology; no reporting by ulcer type \\
\hline Strömberg $1984^{\mathrm{w} 172}$ & Includes ulcers of mixed aetiology; no reporting by ulcer type \\
\hline Teepe $1993^{\text {w173 }}$ & Study examining skin grafts \\
\hline Thomas $1988^{\text {w174 }}$ & Review article \\
\hline Thomas $1989^{\text {w175 }}$ & Includes ulcers of mixed aetiology; no reporting by ulcer type \\
\hline Thomas $1997^{\text {w176 }}$ & Includes ulcers of mixed aetiology; no reporting by ulcer type \\
\hline Torres $2003^{\text {w177 }}$ & Non-randomised study \\
\hline Tosti $1983^{\text {w178 }}$ & Unclear if a randomised controlled trial; no reply to query \\
\hline Varghese $1986^{\text {w179 }}$ & Non-randomised study \\
\hline Viamontes $2003^{\text {w180 }}$ & Non-randomised study \\
\hline Vin $1997^{\text {w181 }}$ & Includes ulcers of mixed aetiology; no reporting by ulcer type \\
\hline Warburg $1994^{\mathrm{w} 182}$ & Study of skin grafting \\
\hline Watts $1988^{\text {w183 }}$ & Includes ulcers of mixed aetiology; no reporting by ulcer type \\
\hline Watts $1993^{\text {w184 }}$ & Study of pressure ulcers and cavity wounds \\
\hline Wayman $2000^{\text {w185 }}$ & No reporting of ulcer healing \\
\hline Westerhof $1990^{\text {w186 }}$ & No reporting of ulcer healing \\
\hline Westerhoff $1993^{\text {w187 }}$ & No reporting of ulcer healing \\
\hline Westerhoff $1995^{\text {w188 }}$ & Report of imaging technique \\
\hline Westh $1998^{\text {w189 }}$ & No reporting of ulcer healing \\
\hline Williams $1981^{\text {w190 }}$ & Study of pilonidal sinus \\
\hline Winter 1990a ${ }^{\text {w191 }}$ & Includes ulcers of mixed aetiology; no reporting by ulcer type \\
\hline Winter $1990 b^{\text {w192 }}$ & Includes ulcers of mixed aetiology; no reporting by ulcer type \\
\hline Wollina $1997^{\text {w193 }}$ & Includes ulcers of mixed aetiology; no reporting by ulcer type \\
\hline Worsley $1991^{\text {w194 }}$ & Includes ulcers of mixed aetiology; no reporting by ulcer type \\
\hline Zeegelaar 1994 & No reporting of ulcer healing \\
\hline Zeegelaar 2001 ${ }^{\text {w196 }}$ & Non-randomised study \\
\hline
\end{tabular}

\section{Hydrogel versus miscellaneous dressings}

One trial (53 participants) consisted of two parts. ${ }^{\text {w38 }}$ Part 1 compared $0.9 \%$ normal saline with Debrisan paste. This phase lasted two weeks, and the authors reported relative change in wound area. Part 2 of the study compared porcine skin (Skin-tec, Astra-Syntex, Sweden), aluminium foil dressing (Metallina, Lohmann $\mathrm{GmbH}$, Germany), and a non-compressive double layer paste bandage. The authors gave no data on the total number of ulcers healed; they reported mean relative reduction in wound area.

\section{Alginate dressings}

\section{Alginate versus low adherent dressings}

One trial (60 participants) compared alginate with low adherent dressings. ${ }^{\text {w39 }}$ It found no statistically significant difference between the two dressings (26/30 healing in the alginate group compared with 24/30 in the low adherent group; relative risk=1.08, 0.86 to 1.36 ).

\section{Alginate versus alginate}

One trial (20 participants) compared different alginate dressings (Tegagen HG and Sorbsan). ${ }^{\mathrm{w} 40}$ The study reported the total number of ulcers healed at eight weeks and found no statistically significant differences between the two groups (relative risk for healing with Tegagen $=0.1,0.01$ to 1.86 ).

Three trials compared alginates with

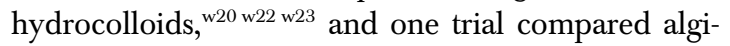
nate with a foam dressing. ${ }^{\text {w34 }}$ These are summarised in the hydrocolloid and foam sections.

One trial (113 patients with 133 ulcerated limbs) compared alginate dressings in a three treatment arm trial. ${ }^{\mathrm{w} 41}$ The allocation was on the basis of the limb and not the patient. The treatments were zinc oxide impregnated cotton bandage (Viscopaste, $n=43$ ), zinc oxide impregnated stockinet (Acoband, $\mathrm{n}=44$ ), and alginate dressing (Kaltostat, $\mathrm{n}=46$ ). The study reported the total number of limbs healed. These were $34 / 43$ 


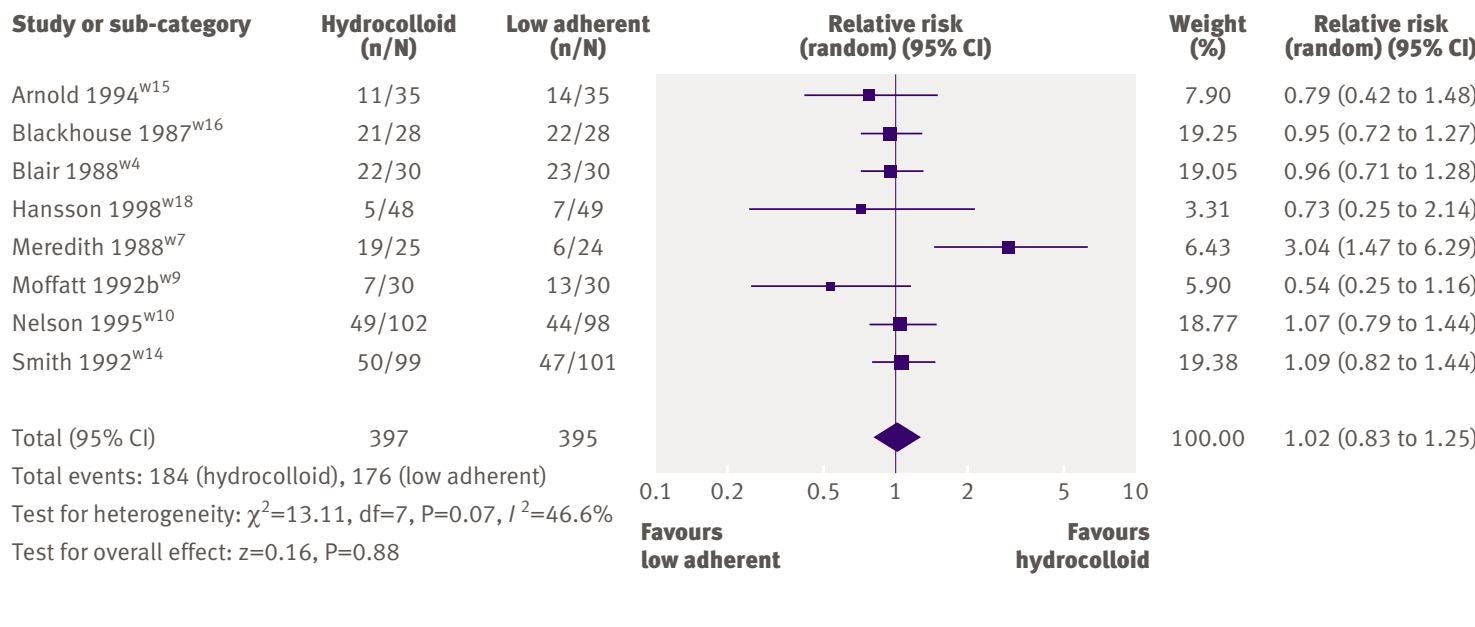

Fig 2 | Hydrocolloid dressings versus low adherent dressings

$(86 \%)$ in the Viscopaste arm, 26/44 (66\%) in the Acoband arm, and 26/46 (57\%) in the alginate arm. The relative risk of healing with the zinc paste bandage compared with the alginate dressing was 0.82 (0.61 to 1.1$)$.

\section{Miscellaneous dressings}

One trial compared a cadoximer iodine powder and "standard" treatment as determined by the clinician. ${ }^{\mathrm{w} 42}$ The trial reported a mean percentage ulcer reduction of $34 \%$ in the cadoximer group compared with $5 \%$ in the standard group.

One trial compared a hyaluronan derivative fleece dressing with paraffin gauze dressing. ${ }^{\text {w43 }}$ It found no statistically significant difference in the numbers of ulcers healed. Two $(16 \%)$ of 12 ulcers healed in eight weeks in the fleece dressing group compared with $1 / 12$ $(8 \%)$ in the paraffin gauze group $\left(\chi^{2}=0.39, \mathrm{df}=1\right.$; $\mathrm{P}=0.53)$. It also reported a statistically significant reduction in ulcer area $-8.1 \mathrm{~cm}^{2}$ for the fleece dressing compared with $0.4 \mathrm{~cm}^{2}$ for paraffin gauze $(\mathrm{P}<0.002)$.

One trial compared a polyamide active charcoal dressing with a dressing applied "according to the stage of healing." ${ }^{{ }^{4} 44}$ It found no statistically significant difference in the numbers healed at six weeks: $6 / 19$ $(31.5 \%)$ in the charcoal dressing group compared with $2 / 20(10.5 \%)$ for the alternative $\left(\chi^{2}=2.78, \mathrm{df}=1\right.$; $\mathrm{P}=0.095)$.

\section{DISCUSSION}

This review updates a systematic review published in $1999^{4}$ and a more limited review published in $2005,{ }^{10}$ which included only 16 studies, compared with 42 in our review. The results from our meta-analysis showed no statistically significant difference in terms of total ulcers healed between any of the dressing types. In some cases this may be due to low power to detect a difference. The meta-analysis of hydrocolloid dressings versus low adherent dressings had more than 700 participants. This means that we can be confident that hydrocolloids confer no significant clinical benefit over simple, low adherent dressings when used beneath compression. Given the potential disadvantages of using hydrocolloid rather than a low adherent simple dressing, in terms of increased cost and greater exposure to allergens in the preservative, the simple non-adherent dressing should be preferred.

One consideration when reviewing these results must be that an intention to treat perspective was used, which assumed that losses to follow-up failed to heal. This could have underestimated the healing rates.

Quality of included trials

Most of the trials included in this review had a small sample size (range 13-200, mean 76, median 70) and therefore had low power to detect clinically important differences. Only one study reported an a priori sample size calculation. ${ }^{\text {w9 }}$ This was a concern, as small trials are at a higher risk of publication bias than large trials. Although the results for healing in these trials were usually inconclusive, and this might indicate that few trials in this area remain unpublished because of the direction of their findings, most trials were not inconclusive in their conclusions. They reported several outcome measures, usually subjective, such as dressing performance (ease of use, patient "comfort"), and often concluded that evidence existed for one dressing performing better than the other.

Most of the trials in this review were funded by dressing manufacturers, and we cannot be certain whether unpublished trials exist, or if, in the published trials, outcome measures have been selectively reported. Each comparison included too few trials to allow a funnel plot to be drawn.

We also found problems in terms of reporting of trials. Only three studies stated the method of randomisation and blinding of allocation, ${ }^{\mathrm{w} 4} \mathrm{w} 10 \mathrm{w} 11$ and only one reported blinding of assessment. ${ }^{\text {w19 }}$ Although blinding of trial participants to many wound dressings is difficult, as they often differ in appearance, blinding of the assessment of treatment can usually be achieved.

The lack of blinding of outcome assessors has implications in the light of a recent study that reported statistically significant differences in the subjective 


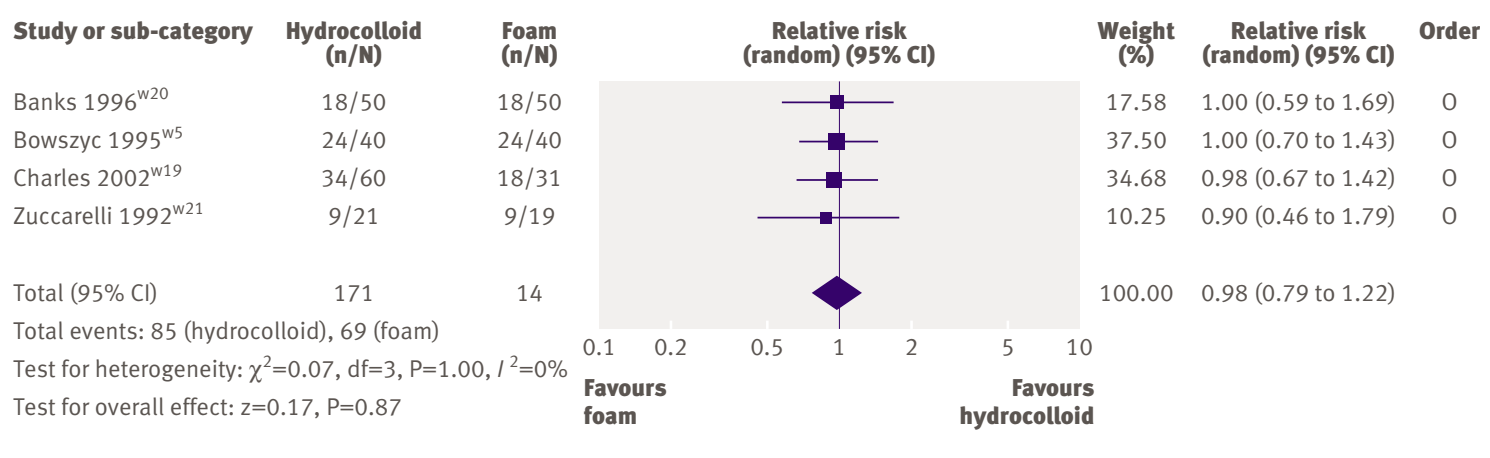

Fig 3 | Hydrocolloid dressings versus foam dressings

assessment of wound progress when the assessors were not blind to the dressing allocated, in contrast to an assessment by blinded outcome assessors in which no difference in wound progress was found. ${ }^{11}$ Evidence also exists to suggest that inadequate methodological reporting has an association with overestimation of treatment effects. ${ }^{12}$

The trials were of relatively short duration (range 448 , mean 14 , median 8 weeks). Venous ulcers usually take months to heal, ${ }^{13}$ so trials with short durations fail to capture most healing events, further eroding power to detect clinically important differences as statistically significant.

\section{External validity of included trials}

The external validity of many of these trials is threatened by the fact that they limited inclusion by ulcer size. Of the 23 studies that reported details of baseline ulcer area, 15 included only ulcers of less than $10 \mathrm{~cm}^{2}$ and eight included only ulcers of greater than $10 \mathrm{~cm}^{2}$. Only one trial used life table analysis, summarising both how many people's ulcers healed and how quickly they healed. ${ }^{\mathrm{w} 9}$ Many studies used rate of reduction in ulcer area as an outcome measure; however, this is not necessarily a predictor of healing, particularly when used over a short period. In addition, the use of change in wound area raises questions of validity, especially when initial ulcer size varies, as the percentage change will be greater for smaller wounds. The use of rates of reduction in area (often called ulcer healing rates) can therefore be misleading. ${ }^{14}$

\begin{tabular}{|c|c|c|}
\hline Comparison & No of trials (total No of participants) & Pooled relative risk $(95 \% \mathrm{Cl})$ \\
\hline Hydrocolloid $v$ low adherent & 8 (792) (1 trial excluded $\left.{ }^{\mathrm{w} 17}\right)$ & $1.02(0.83$ to 1.25$)$ \\
\hline Hydrocolloid $v$ foam & $4(311)$ & $0.98(0.79$ to 1.22$)$ \\
\hline Hydrocolloid $v$ alginate & $3(80)$ & $0.72(0.15$ to 3.42$)$ \\
\hline Hydrocolloid $v$ hydrocolloid & $3(98)$ & $1.56(0.67$ to 3.63$)$ \\
\hline Foam $v$ low adherent & $2(203)\left(1\right.$ trial excluded $\left.^{\mathrm{w} 12}\right)$ & $1.35(0.93$ to 1.94$)$ \\
\hline Foam $v$ foam & $2(136)$ & $1.2(0.77$ to 1.87$)$ \\
\hline Hydrogel $v$ low adherent & $2(151)$ & 1.53 (0.96 to 2.42$)$ \\
\hline Hydrogel $v$ hydrogel & $2(175)$ & NA \\
\hline
\end{tabular}

Other outcome measures used included patient derived and nurse derived subjective measures such as "satisfaction" and pain. The use of subjective outcomes in trials can lead to bias, especially if the tools used are not tested for reliability and validity and if blinding to treatment allocation is not used. Bias can result from subconscious preferences of treatments by the assessors, patients, or both, or selective reporting of positive outcomes.

\section{Cost and quality of life data}

Cost and quality of life data used in the studies were also generally poor quality or lacking. When quality of life measures were reported they tended to be linear analogue scales or simple Likert-type scales. The inclusion of more sophisticated measures of quality of life when evaluating dressings is an area that needs to be tackled. This is particularly important as it may be one of the few ways to distinguish between dressings. The impact of venous ulcers on quality of life has been studied, ${ }^{15-20}$ but within randomised controlled trials quality of life data were very poor or omitted altogether.

The poor reporting of cost data was a particular concern. Where such data were collected, ${ }^{\text {w7w14 w29 the }}$ reporting did not conform to rigorous guidelines for economic evaluations. ${ }^{21}$ The trials simply totalled the monetary cost of the dressings and did not examine their cost effectiveness. This was illustrated in the hydrocolloid versus alginate comparison, where costs were reported for the interventions but insufficient detail was provided on their derivation.

\section{Clinical implications}

Although a wide variety of dressings are available, and used on venous leg ulcers, we found insufficient evidence to justify the use of a particular dressing or dressing type in preference to any other. In particular, the use of hydrocolloid dressings rather than simple, low adherent dressings should be questioned. In the absence of clear evidence of differences in clinical effectiveness, the optimum use of resources demands that the least expensive dressing should be used, although the preferences of patients and nurses may be important where little difference in cost exists. 


\section{WHAT IS ALREADY KNOWN ON THIS TOPIC}

Dressings are applied over ulcers with a view to aiding healing and improving patients' comfort

A wide variety of brands and types of dressing are available, but the evidence for their effectiveness is equivocal

\section{WHAT THIS STUDY ADDS}

Insufficient evidence of effectiveness exists to recommend one type of dressing in preference to another

Hydrocolloid dressings offer no healing benefit compared with simple dressings under compression

In the absence of evidence for healing benefit, cost should be a factor in the choice of dressings
4 Bradley M, Cullum N, Nelson EA, Petticrew M, Sheldon T, Torgerson D. Systematic reviews of wound care management: (2) dressings and topical agents used in the healing of chronic wounds. Health Technol Assess 1999;3(17 II):iii-126.

5 British Medical Association, Royal Pharmaceutical Society of Great Britain. British national formulary. London: BMA, RPS, 2007. (No 53. )

6 Palfreyman SJ, Nelson EA, Lochiel R, Michaels JA. Dressings for healing venous leg ulcers. Cochrane Database Syst Rev 2006;(3): CD001103.

$7 \quad$ NHS Centre for Reviews and Dissemination. Undertaking systematic reviews of research on effectiveness: CRD guidelines for those carrying out or commissioning reviews. York: York Publishing Services, 2001. (CRD Report 4.)

8 Higgins JPT, Thompson SG. Quantifying heterogeneity in a metaanalysis. Stat Med 2002;21:1539-58.

9 QUOROM Group. Improving the quality of reports of meta-analyses of randomised controlled trials: the QUOROM statement. Lancet 1999;354:1896-900.

10 Bouza C, Muñoz A, María J. Efficacy of modern dressings in the treatment of leg ulcers: a systematic review. Wound Repair Regen 2005;13:218-29.

11 Reynolds T, Russell L, Deeth M, Jones H, Birchall L. A randomised controlled trial comparing Drawtex with standard dressings for exuding wounds. I Wound Care 2004;14:71.

Cost effectiveness studies examining dressings for venous leg ulcers are urgently needed, as dressing frequency drives costs by influencing the amount of time taken by clinicians to treat ulcers.

Contributors: SP and EAN reviewed articles and extracted and analysed the data. JAM reviewed articles and advised on inclusion. All authors contributed to drafting the article. SP is the guarantor.

Funding: NHS HTAP, Sheffield Teaching Hospitals Trust, University of York, University of Leeds. The opinions and conclusions expressed here are those of the authors and do not necessarily reflect those of the UK National Health Service or the Department of Health.

Competing interests: A trial by EAN was included in the review.

Ethical approval: Not needed.

Provenance and peer review: Non-commissioned; externally peer reviewed.

1 Cullum N, Nelson EA, Fletcher A, Sheldon T. Compression for venous leg ulcers. Cochrane Database Syst Rev 2001;(2):CD000265.

2 Fletcher A, Cullum N, Sheldon TA. A systematic review of compression treatment for venous leg ulcers. $B M$ J 1997;315:576-80.

3 Palfreyman SJ, Lochiel R, Michaels JA. A systematic review of compression therapy for venous leg ulcers. Vasc Med 1998;3:301-13. 